\title{
Recurrence of herpes zoster in a young woman with IgG3 deficiency
}

\author{
Ki-Hwan Yang, Ki-Ho Mun, Gun Woo Kim, Choon Soo Lee, and \\ Young-Deog Cha
}

Department of Anesthesiology and Pain Medicine, Inha University School of Medicine, Incheon, Korea

Recurrent herpes zoster occurs at a low frequency among immunocompetent individuals. Particularly in young people, recurrent herpes zoster is known to be rare [1]. As the authors found a case of a young woman with no particular past medical history in whom herpes zoster recurred and IgG subclass deficiency was diagnosed on additional testings, we herein report the case.

The patient was a 32-year-old woman who visited the Department of Pain Medicine after having been diagnosed with post-herpetic neuralgia. A year and two months before she visited our hospital, she had experienced symptoms including erythema, grouped vesicles, and piercing pains that began at the part just above the nipple (T3 dermatome) on the left chest. She was diagnosed with herpes zoster, which was treated and cured completely with no apparent sequelae. Six weeks prior to her visit to our hospital, however, the erythema and pains recurred at the same site, and the dermatological clinic diagnosed it as herpes zoster. After the administration of an anti-viral agent for a week and 1,800 mg of gabapentin for six weeks, the skin lesion resolved without leaving a scar, but allodynia, paresthesia, and hyperalgesia continued at the site and, therefore, the patient visited the present hospital and was diagnosed with post-herpetic neuralgia. The patient had been suspected of suffering from narcolepsy, but had not received any treatment. She had no previous medical history of hypertension, diabetes, frequent upper

Corresponding author: Young-Deog Cha, M.D., Ph.D.

Department of Anesthesiology and Pain Medicine, Inha University School of Medicine, 27, Inhang-ro, Jung-gu, Incheon 22332, Korea

Tel: 82-32-890-3968, Fax: 82-32-881-2476

E-mail: ydchaan@inha.ac.kr

Received: June 15, 2015.

Revised: 1st, July 21, 2015; 2nd, July 23, 2015.

Accepted: July 25, 2015.

Korean J Anesthesiol 2015 December 68(6): 622-623 http://dx.doi.org/10.4097/kjae.2015.68.6.622 respiratory infections, or other infectious diseases.

As it was rare that herpes zoster recurred at the same site in a young patient with no particular past medical history, the patient was examined for the presence of an immune disorder. She underwent tests including those for immunoglobulin G (IgG) subclass and anti-nuclear antibody, IgG, IgA, IgM, IgD, vitamin D3, and human immunodeficiency virus (HIV). The laboratory tests revealed, IgG3 levels as low as $20.2 \mathrm{mg} / \mathrm{dl}$ (normal range 21.8-176.1 mg/dl) but there were no other specific findings. Six months later, her testing showed an IgG3 level of $16.0 \mathrm{mg} / \mathrm{dl}$. The case was therefore diagnosed as IgG3 deficiency. The patient was administered herpes zoster vaccine once, and it was confirmed after six months that antibody had not been formed.

Our treatment goals should be focused on restoration of normal function, improved quality of life, reduction of the use of medication, and prevention of relapse of chronic symptoms. Thus, the patient is currently being treated for post-herpetic neuralgia through drug treatment (pregabalin, tramadol + acetaminophen) and two epidural blocks per week. The patient's Numeric Rating Scale score decreases from 8-9 before treatment to 3-4 after, but it rebounds to the original level within 2-3 days after treatment, and she continues to feel pains. She is under follow-up for the IgG3 deficiency.

Herpes zoster is caused by the reactivation of the varicellazoster virus (VZV). In the first infection with VZV, the virus infects $\mathrm{T}$ cells in the tonsils, and becomes latent in ganglia through the infected skin or T cells. The reactivation of the virus latent in ganglia is inhibited by cell-mediated immunity [2]. As individuals grow old, their immunological competence decreases, and if immunological competence drops below a certain threshold, VZV is reactivated. Through axonal transport, the reactivated virus moves through ganglia and symptoms appear in the skin. Among young people as well, herpes zoster may occur when immunological competence has decreased as a result of stress, cancer, HIV infection, etc [2]. Glesby et al. [3] reported that in HIV patients, the recurrence rate of herpes zoster was about

(c) This is an open-access article distributed under the terms of the Creative Commons Attribution Non-Commercial License (http://creativecommons.org/ licenses/by-nc/4.0/), which permits unrestricted non-commercial use, distribution, and reproduction in any medium, provided the original work is properly cited. 
$12 \%$ within a year. The incidence of complications associated with herpes zoster also increases, and the most common one is postherpetic neuralgia, which occurs in $18 \%$ of patients with recurrent herpes zoster [3]. In the case of the patient described here, as the cause of recurrent herpes zoster was not found in her past history, an immunological problem was suspected and tests were done to examine the immune system, to detect the presence of HIV, etc. The testing revealed no sign of infection, including HIV infection, but the levels of IgG3 were found to be significantly low.

The reason for the occurrence of IgG subclass deficiency has not been explained clearly, but genetic problems are found in some patients. Some studies observed a decrease in IgG3 when the allotype of IgG1 increased in patients with IgG subclass deficiency, and some patients showed an increase in the phenotype of IgG3 allotype [4]. However, the definite mechanism has not been discovered yet .

Major clinical symptoms of IgG3 deficiency are upper respiratory infections such as asthma, chronic sinusitis, and pharyngitis at a young age. Severe cases may be accompanied by bacterial pneumonia or chronic bronchitis [5]. In most cases, however, such symptoms disappear along with growth to adulthood. IgG3 deficiency may be asymptomatic and pass un- noticed. In the case of the patient described here, as well, IgG3 deficiency had been unnoticed because there had not been any respiratory symptom such as frequent upper respiratory tract infections, asthma, or sinusitis.

In general, asymptomatic IgG3 deficiency does not require treatment, but if the disease shows symptoms such as frequent upper respiratory infections it should be treated in some way, such as by the administration of immunoglobulin. In the case of asymptomatic IgG3 deficiency, however, it has not been established whether the disorder should be treated or not and even whether it has clinical significance or not [5]. In case of this patient, herpes zoster vaccine was administered but antibody was not formed. Because there are no other symptoms except the recurrence of herpes zoster, we have decided to continue outpatient follow-up with examination and vaccination at the Department of Infectious Diseases.

It is rare that herpes zoster recurs in a young patient without any specific medical history, as in the case discussed here. If herpes zoster recurs in such a young patient, as in this case, it is necessary to investigate for other possible underlying diseases and especially for causes that may affect the patient's immune system. If an immune disorder is found, appropriate therapies should be applied as needed.

\section{References}

1. Donahue JG, Choo PW, Manson JE, Platt R. The incidence of herpes zoster. Arch Intern Med 1995; 155: 1605-9.

2. Kost RG, Straus SE. Postherpetic neuralgia-pathogenesis, treatment, and prevention. N Engl J Med 1996; 335: 32-42.

3. Glesby MJ, Moore RD, Chaisson RE. Clinical spectrum of herpes zoster in adults infected with human immunodeficiency virus. Clin Infect Dis $1995 ; 21: 370-5$.

4. Pan Q, Hammarström L. Molecular basis of IgG subclass deficiency. Immunol Rev 2000; 178: 99-110.

5. Meyts I, Bossuyt X, Proesmans M, De B. Isolated IgG3 deficiency in children: to treat or not to treat? Case presentation and review of the literature. Pediatr Allergy Immunol 2006; 17: 544-50. 